\title{
Slow breathing improves cardiovascular reactivity to mental stress and health-related quality of life in heart failure patients with reduced ejection fraction
}

\author{
Kamila Lachowska ${ }^{1}$, Jerzy Bellwon ${ }^{1}$, Joanna Moryś ${ }^{2}$, Marcin Gruchała ${ }^{1}$, Dagmara Hering ${ }^{1,3}$ \\ ${ }^{1} 1^{\text {st }}$ Department of Cardiology, Medical University of Gdansk, Poland \\ ${ }^{2}$ Department of Clinical Psychology, Medical University of Gdansk, Poland \\ ${ }^{3}$ Department of Hypertension and Diabetology, Medical University of Gdansk, Poland
}

\begin{abstract}
Background: Previous studies have demonstrated therapeutic benefits of slow breathing (SLOWB) in chronic heart failure (HF) but its impact on cardiovascular reactivity in response to laboratory stressors remains unknown.

Methods: Using device-guided breathing this study explored the acute and long-term effects of SLOWB on hemodynamic responses to handgrip, mental and cold pressor tests, and health-related quality of life (QoL) in stable HF patients with reduced ejection fraction (HFrEF) who had received all available optimal drug and device therapies. Blood pressure (BP) and heart rate (HR) were measured in 21 patients with HFrEF (23.9 $\pm 5.9 \%)$ at rest, during laboratory stressors, before and after acute SLOWB, and 12 weeks after SLOWB home training (30 min daily). Health-related QoL (MacNew questionaries) was assessed before and 12 weeks after SLOWB home training.

Results: Resting BP significantly increased in response to three laboratory stressors. Pressor and cardiac changes during mental stress were greater than responses to the handgrip test $(p<0.05)$. Mental stress also produced a greater HR change than cold pressor test $(p<0.05)$. Both acute and long-term $S L O W B$ significantly reduced BP and $H R$ responses to mental stress $(p<0.05)$, but not to isometric and cold pressor tests. SLOWB improved scores of all domains of QoL $(p<0.05)$ at 12 weeks follow-up. Conclusions: These findings demonstrate that SLOWB reduces acute and chronic effects of cardiovascular reactivity to mental stress and improves various aspects of health-related QoL in patients with severe HFrEF. Whether stress reduction and psychological changes achieved with SLOWB may translate to improved outcomes in HFrEF warrants further exploration. (Cardiol J 2020; 27, 6: 772-779)
\end{abstract}

Key words: heart failure with reduced ejection fraction, slow breathing, hemodynamics, laboratory stressors, health-related quality of life

\section{Introduction}

Chronic heart failure (HF) is considered a global pandemic with survival outcomes as malignant as some common cancers [1]. The progression of HF, worsening of symptoms and increasingly fre- quent hospitalizations negatively influence patient functional ability and health-related quality of life (QoL) [2-5]. Depression is one of the most important factors determining QoL in HF patients, this also contributes to social isolation [6,7]. Depressed mood and vital exhaustion are strong predictors of

Address for correspondence: Prof. Dagmara Hering, MD, PhD, Department of Hypertension and Diabetology, Medical University of Gdansk, ul. Dębinki 7c, 80-952 Gdańsk, Poland, tel: +48 58349 2065, fax: +48 583492601 , e-mail: hering@gumed.edu.pl

Received: 22.11.2018 Accepted: 3.01.2019 
low life expectancy, all-cause and cardiovascular (CV) mortality, independent of classical risk factors [8-12]. Acute emotional stress has been linked to a high incidence of acute CV events, arrhythmias and sudden cardiac death [13]. Mental stress per se is a potent trigger for myocardial ischemia. Chronic effects of mental stress and psychological states have been reported as important independent predictors of coronary heart disease, atherosclerosis, hypertension and other adverse consequences [13, 14]. CV responses to mental stress independently predicted fatal and non-fatal cardiac and vascular events in patients with coronary artery disease (CAD) $[15,16]$. Furthermore, altered CV reactivity to acute mental stress were independently associated with an increased risk of earlier mortality in HF [17].

Despite the disease burden, there are a limited number of clinical studies in patients with very severe HF. Given the importance of psychological distress and poor QoL to the severity of $\mathrm{HF}$, associated morbidity and mortality, hospital readmission, and duration of hospital stay [2, 4, 18-21], interventions to specifically target potentiated reactivity to mental challenges, physical exertion [22], social aspects and associated QoL are likely to be of substantial clinical benefit. The feasibility and therapeutic effectiveness of the slow breathing (SLOWB) technique has been documented in chronic HF [23-28]. Very recently, it was documented that SLOWB home training improves clinical symptoms, physical and cardiorespiratory capacity, and vagal activity in patients with severe $\mathrm{HF}$ with reduced ejection fraction ( $\mathrm{HFrEF}$ ) irrespective of HF etiology [29]. However, health-related QoL in response to SLOWB is less known, demonstrating an overall improvement without a clear trend toward a reduction of any specific component [25] or a non-significant tendency toward improved QoL [27]. The impact of SLOWB on CV responses to laboratory stressors remain unknown in HF. Therefore, this study investigated CV reactivity to laboratory-induced stressors and whether, and to what extent home paced SLOWB may restrain hemodynamic responses to challenge stressors and improve QoL in patients with severe HFrEF.

\section{Methods}

\section{Subjects}

The study was approved by the Institutional Ethics Committee and written informed consent was obtained from all patients. This prospective study was previously described in detail [29] and included a total of 21 non-smoking patients (16 males, 5 females) with stable chronic HFrEF diagnosed as per European Society of Cardiology (ESC) guidelines [4, 30]. Patients were recruited from a larger cohort of HF patients following the CONSORT guidelines. Only patients who received all available treatment options including optimal medical drug and device therapies were enrolled. All patients were receiving stable doses of optimal multi-drug therapy which had been kept unchanged for at least 6 weeks prior to study enrolment and was maintained (including drugs and dosage) over the 3-month study period.

\section{Study protocol}

Patients were studied at baseline before and after acute performance of SLOWB exercise, and then at 3 months follow-up of monitored SLOWB home training according to the same protocol. Measurements of continuous beat-to-beat finger blood pressure (BP), heart rate (HR), respiration and saturation (ADInstruments, Dual Bio Amp; ADInstruments, Ltd., Oxford, UK) were recorded in a supine position at rest over a 20-min duration followed by an application of two laboratory stressors (i.e. sustained handgrip, mental arithmetic) under carefully standardized conditions. Then patients performed a SLOWB exercise with the use of device-guided breathing (RESPeRATE ${ }^{\circledR}$ ) for $15 \mathrm{~min}$. Following SLOWB and a 20 -min recovery period, application of sustained handgrip and mental arithmetic tests were repeated. A cold pressor test was performed once at the end of the study protocol, due to its sustained hemodynamic effects. The protocol investigating the impact of SLOWB on CV responses to laboratory stressors is consistent with a previous study in patients with untreated essential hypertension [31].

In line with our established protocol [31-33], an isometric handgrip test was conducted by asking patients to sustain a handgrip of $30 \%$ of patient maximum voluntary contraction using a dynamometer. Mental stress was performed by asking the subject to do serial subtractions as fast as possible. Each stress test lasted $3 \mathrm{~min}$ with a 10 -min rest period between tests. Following a 10-min recovery period, a cold pressor test was performed by immersing the hand into an ice water container for $2 \mathrm{~min}$.

\section{MacNew Health-related Quality of Life questionnaire}

The MacNew is a self-administered heart disease-specific health-related QoL instrument and 
addresses three major QoL domains: the Emotional, Physical, and Social domains. This instrument consists of 27 items, each with a 7 -point Likert response scale, measuring the 3 inter-related domains of physical activities (13-item), emotional (14-item) and social functioning (13-item). There are 5 items related to patient symptoms including angina, shortness of breath, fatigue, dizziness and aching legs. The maximum possible score in any domain is 7 and the minimum is 1 with the minimal important difference of 0.50 points on the 7 -point MacNew global scale and each subscale. Domain scores are calculated by taking the average of the responses to the items in each domain and by averaging all 27 items providing a global health-related QoL score. Amongst other conditions, the MacNew questionnaire has been validated in $\mathrm{HF}$ patients $[34,35]$. As part of the international HeartQOL Study, the MacNew form was translated into Polish demonstrating its reliability and validity in patients with CAD, myocardial infarction and HF [36, 37].

The MacNew questionnaire was completed by all patients at baseline and by $16(76 \%)$ participants 3 months after SLOWB home training.

\section{Serum biochemistry}

Routine blood tests were performed in all patients at each study visit at the associated Clinical Laboratory Centre.

\section{Slow-breathing technique}

A device-guided SLOWB pacing (goal below 10 breaths per minute) was performed twice daily with each session lasting $15 \mathrm{~min}$, through use of an ad hoc device RESPeRATE ${ }^{\circledR}$ (Intercure Ltd., Northern Industrial Area, Israel) as described previously [31]. At the first visit, patients were instructed on how to synchronize their breathing with guiding tones generated by the RESPeRATE ${ }^{\circledR}$ in response to their breathing pattern. Following the completion of all tests at baseline, all patients received the device and a translated training manual on its use in a home setting. Performance of SLOWB exercise was scheduled for two 15-min daily sessions (in total 30 min per day) over the 3 months. Patients were asked to breathe effortlessly and gradually at home, irrespective of the time of the day, in a quiet room and in a comfortable position as recommended in the RESPeRATE ${ }^{\circledR}$ manual. Patients were called by an investigator (K.L.) weekly through the duration of the study to obtain information stored on the device regarding the SLOWB exercise (i.e. number of sessions, therapeutic minutes, initial breathing, final breathing rate, the ability to syn-
Table 1. Heart failure (HF) etiology and associated co-morbidities of the entire study cohort.

\begin{tabular}{lc}
\hline Parameter & Number $(\mathbf{n}=\mathbf{2 1})$ \\
\hline Ischemic HF & $9(43 \%)$ \\
Non-ischemic HF & $12(57 \%)$ \\
Myocardial infarction & $8(38 \%)$ \\
Paroxysmal AF & $3(14 \%)$ \\
Persistent AF & $2(10 \%)$ \\
Arterial hypertension & $8(38 \%)$ \\
Diabetes & $5(24 \%)$ \\
CKD (eGFR $\left.<60 \mathrm{~mL} / \mathrm{min} / 1.73 \mathrm{~m}^{2}\right)$ & $6(29 \%)$ \\
PCI & $6(29 \%)$ \\
CABG & $3(14 \%)$ \\
ICD & $9(43 \%)$ \\
CRT-D & $7(33 \%)$ \\
\hline
\end{tabular}

Data expressed as numbers or percentage (\%). AF - atrial fibrillation; CKD - chronic kidney disease; $\mathrm{PCl}$ - percutaneous coronary intervention; CABG - coronary artery bypass graft; ICD - implantable cardioverter-defibrillator; CRT-D — cardiac resynchronization therapy defibrillator

chronize respiratory rate with guiding tones, breath detection). Patients were asked to repeat all tests after 3 months of SLOWB home exercise.

\section{Statistical analysis}

Results are expressed as means \pm standard deviation (SD) or percentage (\%). Changes in variables between baseline and 3 month follow-up were analyzed using a paired t-test. Analysis was performed on ranks for non-Gaussian data. Statistical analysis was performed using SigmaPlot Version 13.0.0.83 (Systat Software, Inc. Leadtools, Dundas Software LTD. Reg. No. 775201235). The sample size and power calculation for this patient cohort had been published previously [29]. An additional analysis revealed that 10 patients would have $80 \%$ power for a paired t-test in detecting an increase in systolic $\mathrm{BP}(\mathrm{SBP})$ in response to mental stress of $3.4 \mathrm{mmHg}$ at the level of significance 0.05 and an estimated SD of 3.4. The present study revealed that 21 patients with a SD of 3.4 and an increase in SBP of $3.4 \mathrm{mmHg}$ had a power of $99.2 \%$. A value of $\mathrm{p}<0.05$ was considered statistically significant.

\section{Results}

Patient demographic characteristics are shown in Table 1. Twenty-one patients with HFrEF in New York Heart Association classes I $(n=5)$, II $(\mathrm{n}=13)$ and III $(\mathrm{n}=3)$ had a mean left ventricular ejection fraction (LVEF) of $23.9 \pm 5.9 \%$, at the 
Table 2. Acute cardiovascular reactivity to mental stress, handgrip and cold pressor tests in heart failure with reduced ejection fraction patients at baseline.

\begin{tabular}{lcccc}
\hline Measurements & Before test & After test & $\Delta$ changes & $P$ \\
\hline Handgrip test & $113 \pm 7$ & $116 \pm 9$ & $3.4 \pm 3.4$ & $<0.001$ \\
SBP $[\mathrm{mmHg}]$ & $88 \pm 5$ & $90 \pm 6$ & $2.1 \pm 2.4$ & $<0.001$ \\
DBP $[\mathrm{mmHg}]$ & $64 \pm 8$ & $66 \pm 6$ & $2.5 \pm 4.1$ & $<0.05$ \\
HR $[\mathrm{bpm}]$ & & & \\
Mental stress & $114 \pm 9$ & $119 \pm 9$ & $5.7 \pm 5.0$ & $<0.0001$ \\
SBP $[\mathrm{mmHg}]$ & $88 \pm 5$ & $92 \pm 6$ & $3.2 \pm 2.4$ & $<0.0001$ \\
DBP $[\mathrm{mmHg}]$ & $65 \pm 6$ & $69 \pm 8$ & $4.8 \pm 6.0$ & $<0.01$ \\
HR $[\mathrm{bpm}]$ & & & & \\
Cold pressor test & $118 \pm 10$ & $121 \pm 13$ & $3.5 \pm 6.7$ & $<0.01$ \\
SBP $[\mathrm{mmHg}]$ & $90 \pm 5$ & $92 \pm 7$ & $1.5 \pm 2.9$ & $<0.05$ \\
DBP [mmHg] & $64 \pm 7$ & $65 \pm 7$ & $0.9 \pm 4.4$ & 0.39 \\
HR [bpm] & & & & \\
\hline
\end{tabular}

Data are means \pm standard deviation. SBP — systolic blood pressure; DBP — diastolic blood pressure; HR — heart rate

age of $52 \pm 17$ years and a body mass index (BMI) of $28 \pm 4 \mathrm{~kg} / \mathrm{m}^{2}$.

Patients were on optimal drug therapy including beta-blockers (carvedilol, bisoprolol or metoprolol), aldosterone antagonists (spironolactone or eplerenone), angiotensin-converting enzyme inhibitors (ACEI, quinapril, ramipril, enalapril or perindopril). Three patients were receiving angiotensin II receptor blockers (valsartan or telmisartan) due to intolerance of ACEI. Other drugs included amiodarone $(n=5)$, ivabradine $(\mathrm{n}=4)$, trimetazidine $(\mathrm{n}=3)$, furosemide $(\mathrm{n}=11)$, torasemide $(n=9)$, statins $(n=14)$ (atorvastatin, rosuvastatin or simvastatin), acetylsalicylic acid $(\mathrm{n}=5)$ and anticoagulants $(\mathrm{n}=12)$ including warfarin, dabigatran or rivaroxaban. No patients were receiving Entresto (sacubitril/valsartan).

Systolic BP and diastolic BP (DBP) significantly increased in response to acute exposure to handgrip, mental and cold pressor tests at baseline visit (Table 2$)$. However, pressor $(5.7 \pm 4.5$ vs. $3.4 \pm 3.4, \mathrm{p}<0.05)$ and cardiac $(4.8 \pm 6.0$ vs. $2.5 \pm 4.1, \mathrm{p}<0.05)$ responses to mental stress were significantly greater than the handgrip test. Mental stress also produced a greater HR change than cold pressor test $(4.8 \pm 6.0$ vs. $0.9 \pm 4.4$, $\mathrm{p}<0.05)$ but not in BP $(5.7 \pm 4.5$ vs. $3.5 \pm 6.7$, $\mathrm{p}=0.13)$. A significant rise in HR was observed following isometric and arithmetic tests but not cold pressor test (Table 2).

Acute effects of SLOWB on SBP, DBP and HR responses to mental and handgrip stress tests are demonstrated in Table 3. BP and HR responses to isometric handgrip tests were similar before and after acute SLOWB (Table 3).

Acute SLOWB significantly reduced BP and HR responses to mental stress (Table 3 ).

Long-term SLOWB home training resulted in further significant attenuation of BP and HR responses to mental tests but had no significant impact on hemodynamic responses to handgrip and cold pressor tests (Table 3 ).

SLOWB home training significantly improved physical, mental and social components, and a global score in HFrEF (Fig. 1).

\section{Discussion}

The novel finding of the present study is that both acute and long-term SLOWB significantly diminishes CV effects of mental stress but not physical stressors in patients with severe HFrEF. This report also documents that, in these HFrEF patients, a 3-month SLOWB home training improves subjective health-related QoL in all domains including physical, mental, social components and a global score.

Recent reports documenting the effectiveness of SLOWB home training in improving oxygen saturation, cardiorespiratory capacity, functional performance, vagal activity, LVEF and reducing pulmonary pressure and breathlessness highlight the important role of breathing frequency in $\mathrm{HF}$ patients [23, 25, 26, 28, 29, 38-40].

High sympathetic excitation is a hallmark of $\mathrm{HF}$ and independent predictor of $\mathrm{CV}$ mortality in 
Table 3. Changes in systolic blood pressure (SBP), diastolic blood pressure (DBP) and heart rate (HR) in responses to handgrip and mental stress tests before and after acute effects of slow breathing (SLOWB) at baseline, and at 3 month follow-up (3MFU) of SLOWB home training in heart failure with reduced ejection fraction patients.

\begin{tabular}{|c|c|c|c|c|c|c|c|}
\hline Measurement & $\begin{array}{c}\text { Before SLOWB } \\
\text { - baseline }\end{array}$ & $\begin{array}{c}\text { After } \\
\text { SLOWB }\end{array}$ & $\mathbf{P}$ & $\begin{array}{c}\text { Before SLOWB } \\
-3 \mathrm{MFU}\end{array}$ & $\begin{array}{c}\text { After } \\
\text { SLOWB }\end{array}$ & $\mathbf{P}$ & $\begin{array}{l}\text { P: baseline } \\
\text { vs. 3MFU }\end{array}$ \\
\hline \multicolumn{8}{|l|}{ Handgrip test } \\
\hline$\Delta \mathrm{SBP}[\mathrm{mmHg}]$ & $3.4 \pm 3.4$ & $3.0 \pm 3.2$ & 0.49 & $2.5 \pm 3.4$ & $2.6 \pm 3.1$ & 0.91 & 0.25 \\
\hline$\Delta \mathrm{DBP}[\mathrm{mmHg}]$ & $2.1 \pm 2.4$ & $2.1 \pm 2.2$ & 0.38 & $1.8 \pm 2.8$ & $2.7 \pm 2.9$ & 0.57 & 0.52 \\
\hline$\Delta \mathrm{HR}[\mathrm{bpm}]$ & $2.5 \pm 4.1$ & $1.8 \pm 3.3$ & 0.70 & $1.0 \pm 7.6$ & $4.0 \pm 5.4$ & 0.14 & 0.97 \\
\hline \multicolumn{8}{|l|}{ Mental stress } \\
\hline$\Delta \mathrm{SBP}[\mathrm{mmHg}]$ & $5.7 \pm 4.5$ & $3.7 \pm 3.1$ & $<0.05$ & $3.4 \pm 4.1$ & $1.3 \pm 3.4$ & $<0.05$ & $<0.05$ \\
\hline$\Delta \mathrm{DBP}[\mathrm{mmHg}]$ & $3.2 \pm 2.4$ & $2.1 \pm 2.1$ & $<0.01$ & $2.2 \pm 2.4$ & $1.5 \pm 1.9$ & 0.17 & $<0.05$ \\
\hline$\Delta \mathrm{HR}[\mathrm{bpm}]$ & $4.8 \pm 6.0$ & $2.8 \pm 5.9$ & $<0.05$ & $2.7 \pm 5.0$ & $2.2 \pm 4.5$ & 0.19 & $<0.05$ \\
\hline \multicolumn{8}{|c|}{ Cold pressor test } \\
\hline$\Delta \mathrm{SBP}[\mathrm{mmHg}]$ & - & $3.5 \pm 6.7$ & - & - & $3.3 \pm 5.8$ & - & 0.93 \\
\hline$\Delta \mathrm{DBP}[\mathrm{mmHg}]$ & - & $1.5 \pm 2.9$ & - & - & $1.5 \pm 3.1$ & - & 0.95 \\
\hline$\Delta \mathrm{HR}[\mathrm{bpm}]$ & - & $0.9 \pm 4.4$ & - & - & $-0.01 \pm 3.5$ & - & 0.29 \\
\hline
\end{tabular}

Data are means \pm standard deviation.

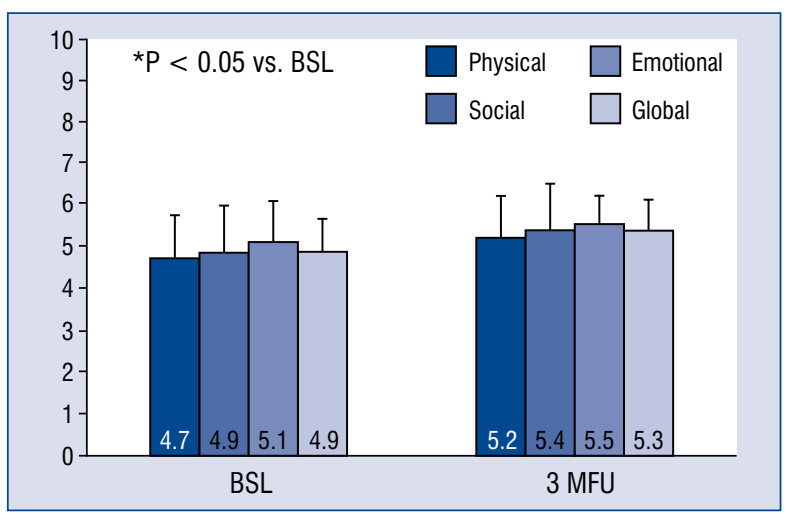

Figure 1. Effects of slow breathing (SLOWB) home training on health-related quality of life changes in physical, mental and social domains, and a global score in heart failure with reduced ejection fraction (HFrEF) patients at 3 month follow-up (3 MFU) compared to baseline (BSL).

chronic HF [41-43]. The magnitude of sympathetic activation has been directly linked to the severity of $\mathrm{HF}$ and reduced left ventricle (LV) function [44]. $\mathrm{LV}$ dysfunction results in several neurohumoral compensatory mechanisms including increases in HR, myocardial oxygen demand, ischemia, myocardial contractility, peripheral vasoconstriction and blood volume to maintain cardiac output and peripheral tissue perfusion [45]. However, with further LV impairment and HF progression, the compensatory regulatory alterations in autonomic control may be counter effective, contributing to a depletion of myocardial noradrenaline (NA) store and decreased myocardial adrenoreceptor density, subsequently potentiating stress to cardiac muscle, impairment of reactivity to exogenous challenges (i.e. physical exertion, fatigue, dyspnoea) and $\mathrm{CV}$ responses, thereby worsening prognosis. While pharmacological blockade of neurohumoral hyperactivation provides a biological rationale for HF management, the increasing number of hospitalizations for HF decompensation and associated death suggests the necessity for additional treatment options. In this context, our recent findings and others of beneficial effects of SLOWB home training are likely to be associated with breaking or weakening the vicious cycle in patients with severe HFrEF [29].

Previous studies have demonstrated that both exaggerated (likely occurring at an earlier stage of disease) and blunted (likely occurring in an advanced stage of disease) CV and hormonal reactivity to psychological stress bear negative prognostic health outcomes [46, 47]. In chronic HF (mean LVEF $31.8 \pm 6.9 \%$ ), low DBP and HR responses to acute mental stress were independently associated with a higher mortality risk [17]. In the present study, patients with severe HFrEF (mean LVEF $23.9 \pm 5.9 \%$ ) had diminished BP and HR reactivity to laboratory stressors compared to patients with untreated essential hypertension [31]. In patients with severe HFrEF, the magnitude 
of pressor and cardiac responses to mental stress were markedly reduced and reached one-fourth and one-third of the values obtained in patients with untreated essential hypertension [31]. Similarly, $\mathrm{CV}$ reactivity to hand-grip and cold pressor tests were substantially attenuated in HFrEF compared to hypertensive patients likely as a result of higher sympathetic drive in the failing heart compared to essential hypertension.

Notably, mental arithmetic stress per se has been shown to induce threefold increases in NA release from sympathetic nerves to the heart in essential hypertension [48] and to cause silent myocardial ischemia in patients with CAD [49]. This observation indicates that repetitive exposure to psychological factors is likely to cause silent damage to the heart, adversely affecting the $\mathrm{CV}$ system (i.e. alterations in myocardial oxygen demand and supply), thereby predisposing acute adverse events. The present study found significant increases in BP and HR responses to all laboratory stressors with a greater $\mathrm{CV}$ reactivity to mental stress than isometric and cold pressor tests. Moreover, in this study acute SLOWB significantly reduced $\mathrm{BP}$ and $\mathrm{HR}$ responses to acute mental stress, whereas a 3-month therapy with SLOWB led to a further and pronounced reduction in $\mathrm{CV}$ responses to mental stress in patients with severe HFrEF that is beyond improvements in oxygen saturation, functional performance levels, cardiorespiratory capacity and HR variability [29]. Whether the incremental benefit on stress reduction achieved with SLOWB is likely to translate to improved outcomes in HFrEF needs to be investigated in larger clinical trials.

A further important finding observed after 3 months of SLOWB was an improvement in health-related QoL including mental, physical, social and global domains. This could be of clinical relevance given the high incidence of depression in $\mathrm{HF}$ and its adverse contribution to the longterm prognosis. The current management of $\mathrm{HF}$ predominantly aims at reducing symptoms of hypervolemia and arrhythmia with the subsequent reduction in hospitalization rates [4], whereas the mental health component is often ignored in clinical practise, mostly due to the lack of the validity and significance of QoL assessment methods. Recently, it has been highlighted that data documenting effects of therapeutic and behavioural interventions on prognostic parameters, exercise performance, functional capacity and psychological status in advance $\mathrm{HF}$ are scarce [3]. Of note, the psychological care is not available for the vast majority of $\mathrm{HF}$ patients and is rather limited to antidepressant therapy [4], indicating an unmet need for patient participation in behavioral therapy to regain control of the disease and manage breathing rate. In this context, the present findings support the notion that the assessment of QoL using MacNew questionnaires can be easily implemented in clinical practice for $\mathrm{HF}$ management, particularly in the absence of a professional psychotherapist. Certainly, SLOWB therapy is easy to use in home settings and a cost-effective approach to be offered to $\mathrm{HF}$ patients to improve patient engagement in health care and associated QoL.

It is noteworthy to mention that a substantial proportion of HF patients on optimal pharmacological and HF device therapies report subjective perceptions of dyspnoea in the absence of objective indications of hypervolemia. In this clinical scenario, SLOWB home training exerts beneficial effects in treating breathlessness. The present findings are supported by objective evidence documenting long-term effectiveness of SLOWB home training in improving oxygen saturation, functional performance levels, cardiorespiratory function and slowing progression of the disease in patients with severe HFrEF [29].

Strengths of the present study include precise evaluations of acute and long-term effects of SLOWB on hemodynamic responses to laboratory stressors in patients with severe $\mathrm{HFrEF}$ who received available treatment options as per ESC guidelines. Secondly, medication remained unchanged during the 3-month therapy of SLOWB home training, thereby eliminating potential confounding effects of drug therapy. Thirdly, all patients reached final breathing rate of $6 \pm 1$ breaths/ $/ \mathrm{min}$ at the end of the RESPeRATE ${ }^{\circledR}$ session and significantly reduced a spontaneous breathing rate at 3 months follow-up [29]. Fourthly, no patient presented with active stimulation during exposure to laboratory tests and response dependent stimulation which might have affected results of the HR change under the stressors. Finally, no patient experienced worsening of $\mathrm{HF}$ or other events over the duration of the study indicating clinical benefits achieved with SLOWB therapy.

\section{Limitations of the study}

The limitations of the present study include a modest number of patients and the lack of a control group. However, this study was a logical continuation of previous clinical experience in patients with essential hypertension who underwent a similar study protocol and could thereby 
serve as controls. Moreover, the present study included patients with severe $\mathrm{HFrEF}$ who received evidence-based therapy for $\mathrm{HF}$ and still remain at high risk for HF decompensation, hospital readmission and HF-related mortality. While the possible occurrence of a potential "learning effect", with repetition of applied stressor tests over time could be viewed as a limitation, it was previously shown that no changes in hemodynamics were observed in response to repeated mental or isometric tests without SLOWB therapy [31].

\section{Conclusions}

The present results indicate that even in the absence of other available treatment options, behavioural intervention such as SLOWB home training appears to have substantial impact on distress and health-related QoL in severe HFrEF on top of evidence-based HF management. These favourable benefits of SLOWB occurred in addition to improvements in cardiorespiratory capacity, functional performance levels, vagal activity and attenuation of $\mathrm{HF}$ progression. While this study involves patients with very severe $\mathrm{HFrEF}$, it seems that SLOWB as an add-on therapy may provide beneficial effects on mental, social, physical and global components in all HF patients independent of HF etiology. Ideally these benefits could be tested in further clinical studies.

\section{Acknowledgements}

The authors would like to thank to Mrs Wiesława Kucharska for her support with patient and data management.

Funding: This study was supported by Research Fellowship Grant from the European Society of Hypertension (01/2007) and the Statutory Grant (ST-85/2015) of the Medical University of Gdansk, Poland.

\section{Conflict of interest: None declared}

\section{References}

1. Mamas MA, Sperrin M, Watson MC, et al. Do patients have worse outcomes in heart failure than in cancer? A primary care-based cohort study with 10-year follow-up in Scotland. Eur J Heart Fail. 2017; 19(9): 1095-1104, doi: 10.1002/ejhf.822, indexed in Pubmed: 28470962.

2. O'Loughlin C, Murphy NF, Conlon C, et al. Quality of life predicts outcome in a heart failure disease management program. Int $\mathrm{J}$ Cardiol. 2010; 139(1): 60-67, doi: 10.1016/j.ijcard.2008.09.003, indexed in Pubmed: 18851887.
3. Nieminen MS, Dickstein K, Fonseca C, et al. The patient perspective: Quality of life in advanced heart failure with frequent hospitalisations. Int J Cardiol. 2015; 191: 256-264, doi: 10.1016/j. ijcard.2015.04.235, indexed in Pubmed: 25981363.

4. Ponikowski P, Voors AA, Anker SD, et al. 2016 ESC Guidelines for the diagnosis and treatment of acute and chronic heart failure: The Task Force for the diagnosis and treatment of acute and chronic heart failure of the European Society of Cardiology (ESC)Developed with the special contribution of the Heart Failure Association (HFA) of the ESC. Eur Heart J. 2016; 37(27): 2129-2200, doi: 10.1093/eurhearti/ehw128, indexed in Pubmed: 27206819 .

5. Kutyifa V, Rice J, Jones R, et al. Impact of non-cardiovascular disease burden on thirty-day hospital readmission in heart failure patients. Cardiol J. 2018; 25(6): 691-700, doi: 10.5603/CJ.2018.0147, indexed in Pubmed: 30600831.

6. Freedland KE, Carney RM, Rich MW, et al. Effect of depression on prognosis in heart failure. Heart Fail Clin. 2011; 7(1): 11-21, doi: 10.1016/j.hfc.2010.08.003, indexed in Pubmed: 21109204.

7. Mbakwem A, Aina F, Amadi C. -Depression in Patients with Heart Failure: Is Enough Being Done? Card Fail Rev. 2016; 2(2): 110 -112, doi: 10.15420/cfr.2016:21:1, indexed in Pubmed: 28785463.

8. Ladwig KH, Baumert J, Marten-Mittag B, et al. Room for depressed and exhausted mood as a risk predictor for all-cause and cardiovascular mortality beyond the contribution of the classical somatic risk factors in men. Atherosclerosis. 2017; 257: 224-231, doi: 10.1016/j.atherosclerosis.2016.12.003, indexed in Pubmed: 28110940.

9. Chen LH, Li CY, Shieh SM, et al. Predictors of fatigue in patients with heart failure. J Clin Nurs. 2010; 19(11-12): 15881596, doi: 10.1111/j.1365-2702.2010.03218.x, indexed in Pubmed: 20579199.

10. Perez-Moreno AC, Jhund PS, Macdonald MR, et al. Fatigue as a predictor of outcome in patients with heart failure: analysis of CORONA (Controlled Rosuvastatin Multinational Trial in Heart Failure). JACC Heart Fail. 2014; 2(2): 187-197, doi: 10.1016/j. jchf.2014.01.001, indexed in Pubmed: 24720928.

11. Hwang SL, Liao WC, Huang TY. Predictors of quality of life in patients with heart failure. Jpn J Nurs Sci. 2014; 11(4): 290-298, doi: 10.1111/jjns.12034, indexed in Pubmed: 24238344.

12. Kowalczys A, Bohdan M, Gruchała M. Prognostic value of daytime heart rate, blood pressure, their products and quotients in chronic heart failure. Cardiol J. 2017 [Epub ahead of print], doi: 10.5603/CJ.a2017.0130, indexed in Pubmed: 29131282.

13. Hering D, Lachowska K, Schlaich M. Role of the sympathetic nervous system in stress-mediated cardiovascular disease. Curr Hypertens Rep. 2015; 17(10): 80, doi: 10.1007/s11906-015-0594-5, indexed in Pubmed: 26318888.

14. Niklas AA, Flotyńska A, Zdrojewski T, et al. Trends in hypertension prevalence, awareness, treatment, and control among Polish adults 75 years and older during 2007-2014. Cardiol J. 2018; 25(3): 333-344, doi: 10.5603/CJ.a2018.0043, indexed in Pubmed: 29671863.

15. Jiang W, Babyak M, Krantz DS, et al. Mental stress--induced myocardial ischemia and cardiac events. JAMA. 1996; 275(21): 1651-1656, indexed in Pubmed: 8637138.

16. Wei J, Rooks C, Ramadan R, et al. Meta-analysis of mental stressinduced myocardial ischemia and subsequent cardiac events in patients with coronary artery disease. Am J Cardiol. 2014; 114(2): 187-192, doi: 10.1016/j.amjcard.2014.04.022, indexed in Pubmed: 24856319.

17. Kupper N, Denollet J, Widdershoven J, et al. Cardiovascular Reactivity to Mental Stress and Mortality in Patients With Heart Failure. JACC: Heart Fail. 2015; 3(5): 373-382, doi: 10.1016/j. jchf.2014.12.016.

18. Faller H, Störk S, Schowalter M, et al. Is health-related quality of life an independent predictor of survival in patients with 
chronic heart failure? J Psychosom Res. 2007; 63(5): 533-538, doi: 10.1016/j.jpsychores.2007.06.026, indexed in Pubmed: 17980227.

19. Lewis EF, Lamas GA, O'Meara E, et al. CHARM Investigators. Characterization of health-related quality of life in heart failure patients with preserved versus low ejection fraction in CHARM. Eur J Heart Fail. 2007; 9(1): 83-91, doi: 10.1016/j. ejheart.2006.10.012, indexed in Pubmed: 17188020.

20. Kato N, Kinugawa K, Seki S, et al. Quality of life as an independent predictor for cardiac events and death in patients with heart failure. Circ J. 2011; 75(7): 1661-1669, indexed in Pubmed: 21532181.

21. Ramos S, Prata J, Rocha-Gonçalves F, et al. Quality of life predicts survival and hospitalisation in a heart failure portuguese population. Appl Res Qual Life. 2016; 12(1): 35-48, doi: 10.1007/ s11482-016-9449-8.

22. Czubaszewski Ł, Straburzyńska-Lupa A, Migaj J, et al. Comparison of prognostic values of cardiopulmonary and heart rate parameters in exercise testing in men with heart failure. Cardiol J. 2018; 25(6): 701-708, doi: 10.5603/CJ.a2017.0070, indexed in Pubmed: 28612902.

23. Bernardi L, Spadacini G, Bellwon J, et al. Effect of breathing rate on oxygen saturation and exercise performance in chronic heart failure. Lancet. 1998; 351(9112): 1308-1311, doi: 10.1016/S01406736(97)10341-5, indexed in Pubmed: 9643792.

24. Boarin S, Malfatto G, Caldara G, et al. Device-guided home-based slow breathing training in patients with congestive heart failure. Effects on exercise capacity, ventilation and ventricular function. Eur Heart J. 2006; 27: 332-332.

25. Parati G, Malfatto G, Boarin S, et al. Device-guided paced breathing in the home setting: effects on exercise capacity, pulmonary and ventricular function in patients with chronic heart failure: a pilot study. Circ Heart Fail. 2008; 1(3): 178-183, doi: 10.1161/CIRCHEARTFAILURE.108.772640, indexed in Pubmed: 19808287.

26. Ekman I, Kjellström B, Falk K, et al. Impact of device-guided slow breathing on symptoms of chronic heart failure: a randomized, controlled feasibility study. Eur J Heart Fail. 2011; 13(9): 10001005, doi: 10.1093/eurjhf/hfr090, indexed in Pubmed: 21803755.

27. Drozdz T, Bilo G, Debicka-Dabrowska D, et al. Blood pressure changes in patients with chronic heart failure undergoing slow breathing training. Blood Press. 2016; 25(1): 4-10, doi: 10.3109/08037051.2016.1099800, indexed in Pubmed: 26513698.

28. Kawecka-Jaszcz K, Bilo G, Drożdż T, et al. Effects of deviceguided slow breathing training on exercise capacity, cardiac function, and respiratory patterns during sleep in male and female patients with chronic heart failure. Pol Arch Intern Med. 2017; 127(1): 8-15, doi: 10.20452/pamw.3890, indexed in Pubmed: 28075423.

29. Lachowska K, Bellwon J, Narkiewicz K, et al. Long-term effects of device-guided slow breathing in stable heart failure patients with reduced ejection fraction. Clin Res Cardiol. 2019; 108(1): 48-60, doi: 10.1007/s00392-018-1310-7, indexed in Pubmed: 29943271.

30. Ponikowski P, Voors AA, Anker SD, et al. 2016 ESC Guidelines for the diagnosis and treatment of acute and chronic heart failure]. Kardiol Pol. 2016; 74(10): 1037-1147.

31. Hering D, Kucharska W, Kara T, et al. Effects of acute and longterm slow breathing exercise on muscle sympathetic nerve activity in untreated male patients with hypertension. J Hypertens. 2013; 31(4): 739-746, doi: 10.1097/HJH.0b013e32835eb2cf, indexed in Pubmed: 23385649.

32. Hering D, Kara T, Kucharska W, et al. High-normal blood pressure is associated with increased resting sympathetic activity but normal responses to stress tests. Blood Press. 2013; 22(3): 183-187, doi: 10.3109/08037051.2012.759689, indexed in Pubmed: 23356493.

33. Hering D, Kara T, Kucharska W, et al. Longitudinal tracking of muscle sympathetic nerve activity and its relationship with blood pressure in subjects with prehypertension. Blood Press. 2016;
25(3): 184-192, doi: 10.3109/08037051.2015.1121708, indexed in Pubmed: 26654200.

34. Dixon T, Lim LLY, Oldridge NB. The MacNew heart disease health-related quality of life instrument: reference data for users. Qual Life Res. 2002; 11(2): 173-183, indexed in Pubmed: 12018740 .

35. Höfer S, Schmid JP, Frick M, et al. Psychometric properties of the MacNew heart disease health-related quality of life instrument in patients with heart failure. J Eval Clin Pract. 2008; 14(4): 500-506, doi: 10.1111/j.1365-2753.2007.00905.x, indexed in Pubmed: 18462292 .

36. Oldridge N, Saner H, McGee HM. The Euro Cardio-QoL Project. An international study to develop a core heart disease health-related quality of life questionnaire, the HeartQoL. Eur J Cardiovasc Prev Rehabil. 2005; 12(2): 87-94, indexed in Pubmed: 15785293.

37. Moryś JM, Höfer S, Rynkiewicz A, et al. The Polish MacNew heart disease heath-related quality of life questionnaire: a validation study. Cardiol J. 2015; 22(5): 541-550, doi: 10.5603/ CJ.a2015.0027, indexed in Pubmed: 26004936.

38. Bernardi L, Porta C, Spicuzza L, et al. Slow breathing increases arterial baroreflex sensitivity in patients with chronic heart failure. Circulation. 2002; 105(2): 143-145, indexed in Pubmed: 11790690.

39. Kawauchi TS, Umeda II, Braga LM, et al. Is there any benefit using low-intensity inspiratory and peripheral muscle training in heart failure? A randomized clinical trial. Clin Res Cardiol. 2017; 106(9): 676-685, doi: 10.1007/s00392-017-1089-y, indexed in Pubmed: 28255812.

40. Drozdz T, Bilo G, Debicka-Dabrowska D, et al. Blood pressure changes in patients with chronic heart failure undergoing slow breathing training. Blood Press. 2016; 25(1): 4-10, doi: 10.3109/08037051.2016.1099800, indexed in Pubmed: 26513698.

41. Cohn JN, Levine TB, Olivari MT, et al. Plasma norepinephrine as a guide to prognosis in patients with chronic congestive heart failure. N Engl J Med. 1984; 311(13): 819-823, doi: 10.1056/ NEJM198409273111303, indexed in Pubmed: 6382011.

42. Kaye DM, Lefkovits J, Jennings GL, et al. Adverse consequences of high sympathetic nervous activity in the failing human heart. J Am Coll Cardiol. 1995; 26(5): 1257-1263, doi: 10.1016/07351097(95)00332-0, indexed in Pubmed: 7594040.

43. Petersson M, Friberg P, Eisenhofer G, et al. Long-term outcome in relation to renal sympathetic activity in patients with chronic heart failure. Eur Heart J. 2005; 26(9): 906-913, doi: 10.1093/ eurheartj/ehi184, indexed in Pubmed: 15764611.

44. Grassi G, Seravalle G, Cattaneo BM, et al. Sympathetic activation and loss of reflex sympathetic control in mild congestive heart failure. Circulation. 1995; 92(11): 3206-3211, indexed in Pubmed: 7586305.

45. Lucia Cde, Femminella G, Gambino G, et al. Adrenal adrenoceptors in heart failure. Frontiers Physiol. 2014; 5: 246, doi: 10.3389/ fphys.2014.00246.

46. Phillips A. Blunted as well as exaggerated cardiovascular reactivity to stress is associated with negative health outcomes. Jpn Psychol Res. 2011; 53(2): 177-192, doi: 10.1111/j.14685884.2011.00464.x.

47. Phillips AC, Ginty AT, Hughes BM. The other side of the coin: blunted cardiovascular and cortisol reactivity are associated with negative health outcomes. Int J Psychophysiol. 2013; 90(1): 1-7, doi: 10.1016/j.ijpsycho.2013.02.002, indexed in Pubmed: 23454029.

48. Esler M, Jennings G, Lambert G. Measurement of overall and cardiac norepinephrine release into plasma during cognitive challenge. Psychoneuroendocrinology. 1989; 14(6): 477-481, indexed in Pubmed: 2623135.

49. Deanfield JE, Kensett M, Wilson RA, et al. Silent myocardial ischaemia due to mental stress. Lancet. 1984; 2(8410): 1001-1005, indexed in Pubmed: 6149394. 